\title{
LA TENUE LÍNEA DE SEPARACIÓN ENTRE LOS MODOS 2 Y 3 EN EL CAMPO DE GIBRALTAR (SUR DE LA PENÍNSULA IBÉRICA, ESPAÑA). UNA PROPUESTA HISTÓRICA
}

\author{
THE THIN LINE BETWEEN MODES 2 AND 3 IN THE CAMPO DE GIBRALTAR \\ (SOUTHERN IBERIAN PENINSULA, SPAIN). A HISTORIC PROPOSAL
}

\author{
VICENTE CASTAÑEDA FERNÁNDEZ* \\ FRANCISCO TORRES ABRIL** \\ LUIS PÉREZ RAMOS** \\ YOLANDA COSTELA MUÑOZ**
}

\begin{abstract}
Resumen: Este trabajo presenta como objetivo central el estudio geológico y geomorfológico de la terraza marina donde se localizan los yacimientos, el análisis de las materias primas seleccionadas para la fabricación de las herramientas de trabajo por las sociedades del paleolítico (modo 2) y un análisis tecnológico de las industrias localizadas con el objeto de comprender las características propias relacionadas con los procesos técnicos de fabricación de las herramientas de trabajo. Finalmente, se procederá a la realización de una contextualización histórica, donde valoraremos la continuidad del modo 2 al modo 3, indicando sus semejanzas y sus diferencias en el ámbito del sur peninsular durante OIS5.

Palabras claves: Paleolítico, Estrecho de Gibraltar, achelense, modo 2, Campo de Gibraltar.
\end{abstract}

\section{ANTECEDENTES}

La comarca del Campo de Gibraltar se localiza en el extremo Sur de la Península Ibérica, un territorio tremendamente interesante desde un punto de vista geológico, medioambiental e histórico. Todo ello acrecentado si nos encontramos ante la definición de comprender y explicar

\footnotetext{
* Área de Prehistoria. Facultad de Filosofía y Letras. Universidad de Cádiz. Avda. Gómez Ulla, s/n. 11003 Cádiz. e-mail: vicente. castaneda@uca.es

** Miembro del Grupo de Investigación titulado Primeras ocupaciones humanas y sus inferencias socioeconómicas en el extremo Sur de la Península Ibérica (PAI-HUM-831).
}

Abstract: The aim of this article is the geological and geomorphological study of the marine terrace where the sites are located, the mineral raw material selected for tool manufacturing by the societies of the Paleolithic periods (modo 2) and a technological analysis of the industries located there in order to understand the characteristics related to the technical processes of tool manufacturing. Finally, an historical contextualization will be carried out, where the continuity of the modo 2 to the modo 3 will be assessed, highlighting its similarities and differences in the area of the peninsular south during OIS5.

Key words: Palaeolithic, Estrecho de Gibraltar, Achelense, modo 2, Campo de Gibraltar.

su primer poblamiento humano, dada la proximidad geográfica del continente africano, el más dinámico durante el paleolítico y donde se origina la humanidad.

La investigación paleolítica ha sido revalorizada en los últimos años en la comarca del Campo de Gibraltar gracias al desarrollo de distintos proyectos de investigación ${ }^{1}$ e intervenciones puntuales, donde las primeras ocupaciones humanas se remontan probablemente a

1. Aquí tendríamos que mencionar el proyecto de investigación titulado Las bandas de cazadores-recolectores en el Campo de Gibraltar, autorizado y subvencionado por la Consejería de Cultura de la Junta de Andalucía, y que cuenta con la responsabilidad del Prof. Vicente Castañeda (UCA). Igualmente, también tendríamos que 
OIS7, en momentos avanzados del modo 2 (Castañeda, coord. 2008).

La base de este trabajo se centra en el registro arqueológico localizado en superficie en distintas terrazas marinas asociados a OIS5, y donde únicamente se han identificado productos realizados en roca. Para su estudio se ha empleado la formulación dialéctica planteada por el Sistema Lógico Analítico (Carbonell 1982; Carbonell et al. 1983; Carbonell et al. 1992), que se adecuan a nuestros planteamientos teóricos de partida y que ya ha sido expuesta en otras ocasiones (Castañeda et al. 2005).

El estudio de los procesos de reducción lítica y de configuración de las distintas herramientas de trabajo nos permitirá inferir, por una parte, los gestos técnicos empleados tanto de una forma sincrónica como diacrónica; mientras que por otra parte, nos aproxima a los modos de trabajo desarrollados por estas sociedades. Esta información, unida a la localización geográfica de los diferentes sitios y al análisis del territorio circundante, nos ayuda a comprender la ordenación social del territorio, y, en última instancia, a un acercamiento a los modos de vida de estas sociedades.

Las evidencias materiales identificadas con las últimas etapas del modo 2 en el ámbito peninsular y asociadas a OIS5, se relacionan con un proceso de selección y aprovisionamiento de las materias primas, donde progresivamente va aumentando el empleo de un tipo de roca como es el sílex, más acorde con las nuevas estrategias de talla. Así, se observa de una forma paulatina la desaparición de los macroútiles (triédros, bifaces y cantos tallados) en beneficio de las herramientas sobre BP de tamaño mediano/pequeño y la progresiva ausencia de herramientas de gran formato sobre BP (bifaces y triédros). Como podremos comprobar más adelante, la introducción de una nueva materia prima como es el sílex se realiza en proporciones ínfimas en el modelo propuesto para esta época en la comarca del Campo de Gibraltar.

Para este trabajo, nos vamos a centrar en los sitios de Guadalquitón-Borondo (San Roque, Cádiz), Guadalquitón-Borondo-1 (San Roque, Cádiz), Guadalquitón-Borondo-2 (San Roque, Cádiz), Guadalquitón-Borondo-3 (San Roque, Cádiz), Guadalquitón-Borondo-4 (San Roque, Cádiz), La Alcaidesa (San Roque, Cádiz), Cortijo Carrasco (San Roque-La Línea de la Concepción, Cádiz), Torre Nueva (La Línea de la Concepción, Cádiz), Punta Mala (La Línea de la Concepción, Cádiz), Los Partichuelos (La Línea de la Concepción, Cádiz)(Castañeda

mencionar los estudios sobre el Paleolítico desarrollados por F. Giles y C. Finlayson en la comarca, especialmente en el Peñón de Gibraltar. et al., 2008) y Ringo-Trinchera-1 (Los Barrios, Cádiz) (Castañeda, coord., 2008). Todos ellos presentan la característica de estar localizados en depósitos de terrazas marinas, que por su posición cronoestratigráfica se asocian a OIS5, a excepción de Ringo Trinchera-1, situado en una terraza fluvial del río Palmones (Fig. 1).

A pesar del avance arqueográfico que se ha documentado en los últimos años en el estudio de las sociedades del paleolítico en el Campo de Gibraltar, aún son bastante los aspectos a desarrollar. Uno de ellos es, sin duda, la reconstrucción paleoclimatológica y paleoambiental de la comarca entre OIS7 y OIS3, y especialmente en OIS5, momento histórico relacionado con este trabajo.

El modelo paleoclimático de la comarca del Campo de Gibraltar, y en general del extremo Sur de la Península Ibérica, empieza a concretarse para el Pleistoceno gracias a los registros continentales (Finlayson y Giles 1999) y a los sondeos oceánicos (MD95-2042 y MD952043)(Sánchez Goñi y d'Errico 2005). A partir de estas evidencias, y en términos generales, se puede comprobar cómo durante OIS7 y OIS5 se mantienen unas condiciones climáticas templadas parecidas a las actuales, mientras que durante el OIS6 se produce un recrudecimiento de las temperaturas, que se dejará notar especialmente en la cobertura vegetal.

Independientemente de los momentos críticos sufridos durante los períodos glaciales, probablemente los ecosistemas mediterráneos del extremo Sur de la Península Ibérica no se modificaron definitivamente tal como ocurrió en latitudes más elevadas (Ruiz Bustos 1991 y 1997). Así, los estudios relacionados con la macrofauna realizados hasta la fecha no han documentado la presencia de fauna de "clima frío", aunque sí los desarrollados sobre la microfauna, más susceptible a las variaciones paleoclimáticas y paleoecológicas. Por el contrario, las secuencias polínicas regionales si han permitido comprobar modificaciones paleocológicas drásticas, aunque este hecho debería contextualizarse atendiendo a los condicionamientos paleogeográficos y medioambientales existentes en el extremo Sur de la Península Ibérica (orografía, proximidad/distancia al mar, altitud/latitud, pluviosidad,...), que favorecería el desarrollo de "áreas refugio" de determinadas especies vegetales características, tal como ocurre en la actualidad, de un clima más riguroso.

La información paleoclimática y paleoambiental tan sólo se verá ampliada por medio de la investigación regional y comarcal, base necesaria y fundamental para crear un modelo paisajístico característico del extremo Sur de la Península Ibérica durante los momentos finales del Pleistoceno. 


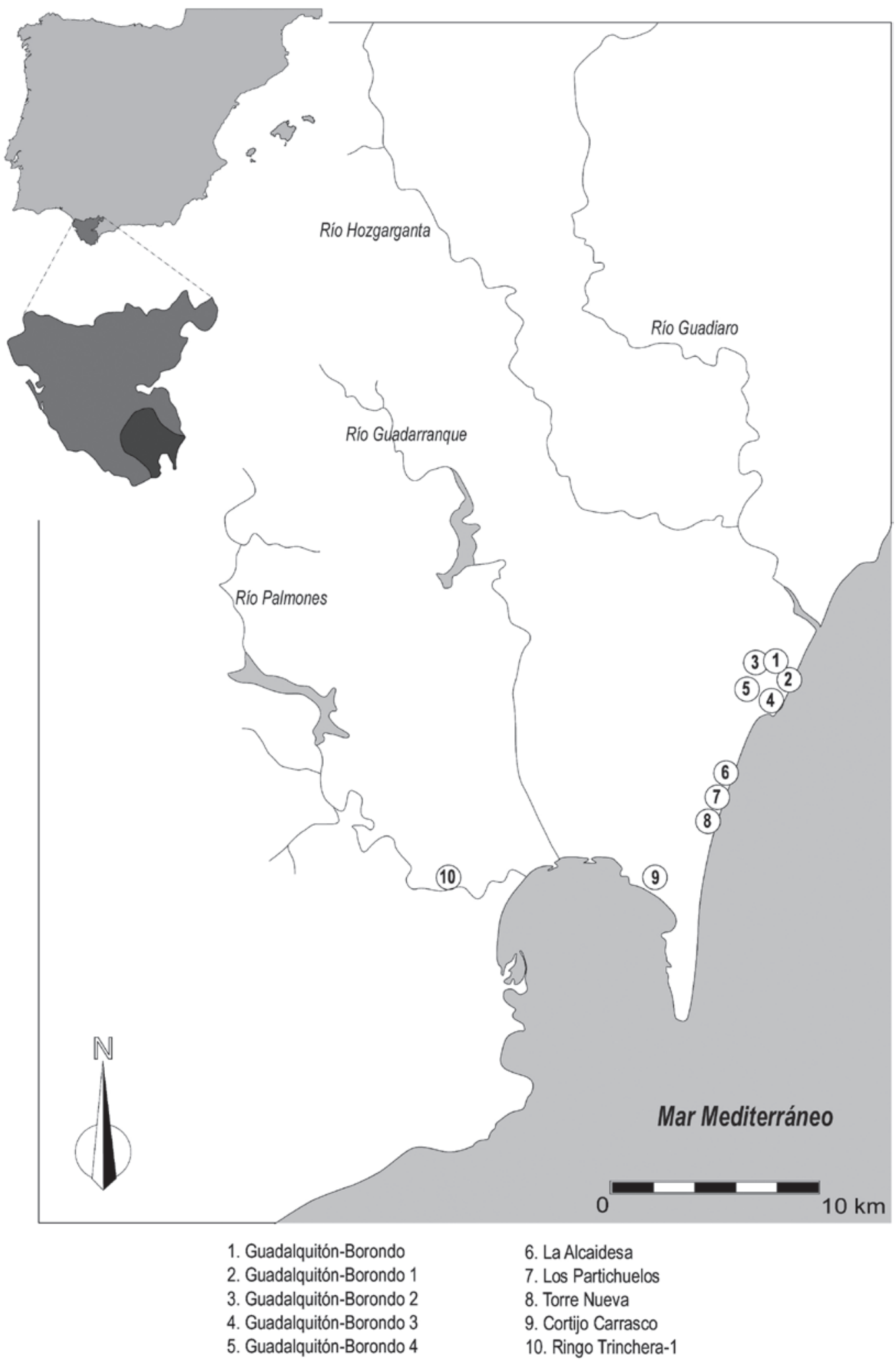

Figura 1. Localización geográfica de los sitios arqueológicos de finales del modo 2 en el Campo de Gibraltar. 


\section{GEOLOGÍA Y GEOMORFOLOGÍA}

Los yacimientos arqueológicos aquí presentados se localizan en la franja litoral de los términos municipales de San Roque y La Línea de la Concepción (Cádiz), en las primeras estribaciones al E y al S de Sierra Carbonera. Esta alineación montañosa costera está formada por potentes paquetes de Arenisca del Aljibe (Gavala 1924; Pendón 1978) estructurados en un manto de cabalgamiento perteneciente a la Unidad Paleogeográfica denominada Surco Turbidítico del Campo de Gibraltar (Martín-Algarra 1988). Nos encontramos en el extremo W de las Cordilleras Béticas, en la estructura conocida como Arco de Gibraltar (Ruiz-Reig., 1994), que une los orógenos alpinos de ambas orillas del Mediterráneo occidental. A la vez, estamos en el contacto entre las placas litosféricas africana y euroasiática, que tienden a converger, lo que ha provocado un levantamiento generalizado de la orilla $\mathrm{N}$ del Estrecho (aunque con excepciones locales donde incluso ha habido subsidencia) de 0,15-0,1 mm/año, para los últimos $128 \mathrm{Ka}$ (Zazo, et al. 1999).

La conjunción de dicha elevación tectónica con las oscilaciones glacioeustáticas del nivel del mar ocurridas durante el Cuaternario, con fuertes descensos durante los periodos glaciales, y excavación de plataformas de abrasión, durante las altas paradas del nivel del mar (highstand) ocurridas en los óptimos interglaciales, sobre las que se depositan sedimentos costeros, ha conformado para nuestra zona una serie de unidades morfosedimentarias escalonadas en el espacio y en el tiempo, con registros marinos erosivo-sedimentarios, que hemos identificado como terrazas marinas $(\mathrm{Tm})$ (Fig. 2) correspondientes a épocas interglaciales en las que el mar estaba a alturas similares o pocos metros por encima del nivel actual.

En el Cuadro 1 se recogen las terrazas marinas reconocidas para nuestra zona de estudio, las cotas a las que se sitúan, así como el tipo de separación que existe entre ellas. Las alturas están referidas al nivel medio de la pleamar (m.a.s.l.).

Cada unidad morfosedimentaria consiste en una plataforma de abrasión, más o menos amplia, donde abundan las bioerosiones y que se corresponde con momentos transgresivos de una alta parada marina. Sobre esta rasa, y durante la etapa regresiva, se depositan secuencias sedimentarias de playa-duna-aluvial.

El material arqueológico recuperado de los distintos yacimientos, durante la campaña de prospecciones superficiales, está en relación con los sedimentos aluviales de la unidad morfosedimentaria $\mathrm{Tm}_{2}$.

La falta de elementos para realizar dataciones absolutas, ya que la fauna fósil es escasísima y está muy alterada, solo nos permite dataciones relativas por comparación con las unidades morfosedimentarias similares descritas en la zona (Giles et al. 2000; Goy et al. 1995; Rodriguez-Vidal et al. 2002; Zazo et al. 1997 y 1999). El estudio geomorfológico y estratigráfico indicaría el nivel marino del último interglacial (estadio isotópico 5) para la $\mathrm{Tm}_{2}$. Ahora bien, la existencia en numerosos puntos del tramo de costa estudiado de gravas cuarzosas de origen litoral, a cotas intermedias entre $\mathrm{Tm}_{1}$ y $\mathrm{Tm}_{2}$ relacionadas o no con una pequeña plataforma, nos lleva a proponer para la zona la presencia de dos momentos de altas paradas del nivel del mar para el estadio isotópico 5 , que, por comparación con las datadas en la región, se corresponderían con los subestadios isotópicos 5 e y 5 c (130-122 y 105-93 ka, respectivamente). Los depósitos marino-dunares de la unidad morfosedimentaria $\mathrm{Tm}_{2}$ corresponderían al subestadio isotópico $5 \mathrm{e}$, mientras que los depósitos de playa entre $\mathrm{Tm}_{1}$ y $\mathrm{Tm}_{2}$ serían asimilables al subestadio isotópico $5 \mathrm{c}$. Según esto, la edad de los depósitos aluviales que fosilizan los sedimentos de terraza marina $\mathrm{Tm}_{2}$, y que contienen los productos arqueológicos, sería poco inferior a $122 \mathrm{Ka}$ (ya que se depositaron en la etapa regresiva inmediatamente posterior al máximo transgresivo del subestadio isotópico 5e). Por último señalar que la $\mathrm{Tm}_{3}$ representaría

Cuadro 1. Terrazas marinas localizadas en los términos municipales de San Roque y La Línea de la Concepción.

\begin{tabular}{|c|c|c|c|c|c|c|}
\cline { 2 - 6 } \multicolumn{1}{c|}{} & Guadalquitón & Punta Mala & Alcaidesa & Los Partichuelos & Torre Nueva & Cortijo Carrasco \\
\hline $\mathbf{T m}_{\mathbf{3}}$ & $+29 \mathrm{~m}$ & $+26 \mathrm{~m}$ & $+24 \mathrm{~m}$ & & $+22 \mathrm{~m}$ & $+23 \mathrm{~m}$ \\
& Abanico aluvial & Abanico aluvial & & Abanico aluvial & Escarpe & Aluvial y escarpe \\
$\mathbf{T m}_{\mathbf{2}}$ & $+11 \mathrm{~m}$ & $+8 \mathrm{~m}$ & Aluvial y escarpe & $+7 \mathrm{~m}$ & $+7 \mathrm{~m}$ & $+11 \mathrm{~m}$ \\
& Escarpe & Abanico aluvial & & Aluvial y escarpe & Escarpe & $?$ \\
$\mathbf{T m}_{\mathbf{1}}$ & $+2,5 \mathrm{~m}$ & $+2 \mathrm{~m}$ & $+2 \mathrm{~m}$ & $+2 \mathrm{~m}$ & $+2 \mathrm{~m}$ & $?$ \\
\hline
\end{tabular}




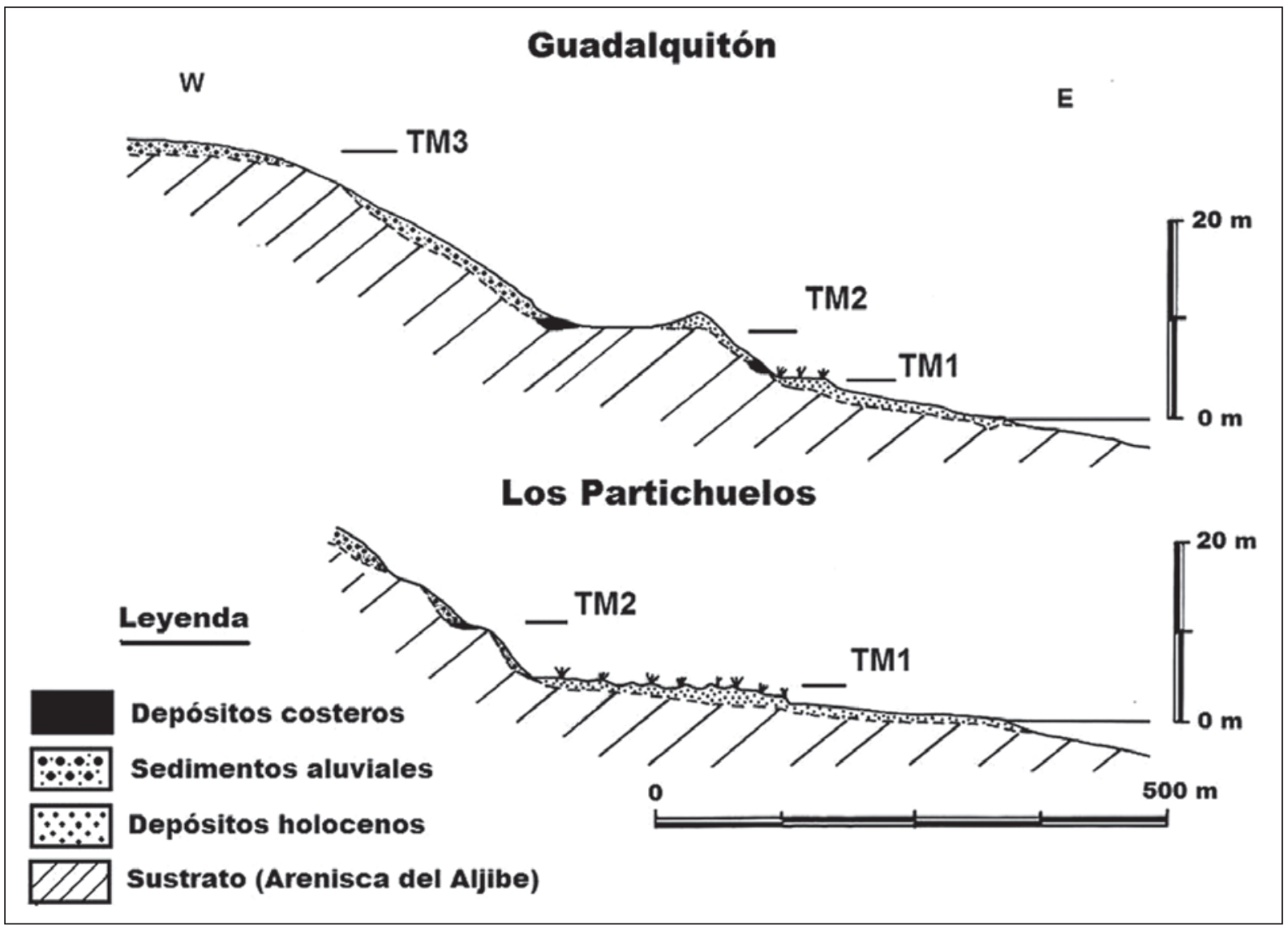

Figura 2. Cortes geológicos de las terrazas marinas de Guadalquiton-Borondo y Los Partichuelos.

alguna de las altas paradas del nivel del mar correspondientes al estadio isotópico 7 (249-187 ka), mientras que la $\mathrm{Tm}_{1}$, claramente holocena, correspondería al máximo transgresivo del presente interglacial, datado en 6500 años antes de presente (Lario 1996).

\section{MATERIAS PRIMAS Y ÁREAS DE CAPTACIÓN}

Los estudios geoarqueológicos realizados han estado enfocados a: la identificación de las materias primas minerales seleccionadas en los procesos de transformación y fabricación de las herramientas de trabajo, determinar la procedencia de las mismas, así como los criterios de selección utilizados.

Para ello se realizó la clasificación mineralógica y petrológica $a v i s u$ de todo el material arqueológico recuperado. Además, se confeccionaron una serie de láminas delgadas de los principales tipos litológicos (tanto del material arqueológico como de las principales rocas aflorantes en la comarca) para su análisis con microscopía de luz polarizada (lám. I) y la contrastación entre los materiales geológicos y arqueológicos, a fin de identificar las posibles fuentes de materias primas líticas durante este periodo.

La práctica totalidad de los restos materiales localizados están elaborados en Arenisca del Aljibe de distintos tamaños de grano. Se trata de una roca con contenidos en cuarzo cercanos al 100\%, muy compacta, cementada principalmente por sílice, y capaz de producir filos cortantes ante los procesos de talla. El uso de las areniscas más duras y compactas indica que el grado de cementación constituye uno de los principales criterios de selección para el aprovisionamiento de materias primas minerales durante este periodo. Otros tipos de rocas como sílex o cuarcitas de origen metamórfico aparecen en proporciones ínfimas.

La distribución porcentual de litologías (Fig. 3), muestra un dominio de las areniscas de grano fino y medio. La comparación de estos datos con sitios arqueológicos más antiguos dentro del modo 2, como Algetares 




Lámina 1. Imágenes mediante microscopía óptica (luz doblemente polarizada) de láminas delgadas realizadas sobre material arqueológico. A) Arenisca de grano muy fino, B) Arenisca de grano medio, C) Arenisca de grano muy grueso, D) Metacuarcita.

(Algeciras, Cádiz)(Castañeda et al. e.p. a), o con sitios más modernos dentro del IV-III milenio a.n.e., como Ventorrillo de la Trocha 1 y 2 (Algeciras, Cádiz) (Castañeda et al. e.p. b), nos lleva a la conclusión de que se produce una disminución en el tamaño de grano de las materias primas minerales detríticas utilizadas a lo largo del tiempo. Pasamos del predominio de las areniscas de grano medio y grueso en sitios adscritos al modo 2, a la mayor abundancia de areniscas de grano fino y medio, pero con importante presencia, aún, de las de grano grueso en los yacimientos de transición entre los modos 2 y 3 , como los que aquí nos ocupan, llegando a la preeminencia de las de grano fino, medio y muy fino en sitios como Ventorrillo de la Trocha 1 y 2 .

Esta disminución en el tamaño de grano de las areniscas a lo largo de la Prehistoria indicaría la necesidad de materias minerales más homogéneas e isótropas, y por tanto con una fractura más precisa ante los procesos de talla, condición necesaria por las técnicas empleadas y el proceso de microlitización de las herramientas de trabajo durante la Prehistoria Reciente. La culminación de este proceso sería la sustitución de la arenisca por el sílex, hecho que en la comarca del Campo de Gibraltar no llega a producirse totalmente, perviviendo el uso de areniscas hasta épocas muy recientes. Las razones de esto habría que buscarlas en la gran escasez de afloramientos de sílex en nuestra zona, lo que fue suplido con la utilización del material existente.

Con respecto a la procedencia de las areniscas utilizadas como materia prima mineral, proponemos un origen local para todas ellas, concretamente los cantos de abanicos aluviales y coluviales tan abundantes en la franja litoral estudiada, donde podrían disponer de nódulos sueltos de diversos tamaños, que provienen de la erosión de Sierra Carbonera y Sierra del Arca. En este sentido hay que destacar la abundancia en estas sierras de estratos muy cementados por sílice y por tanto la riqueza de este tipo de arenisca en los productos de su 


\section{Los Partichue los}

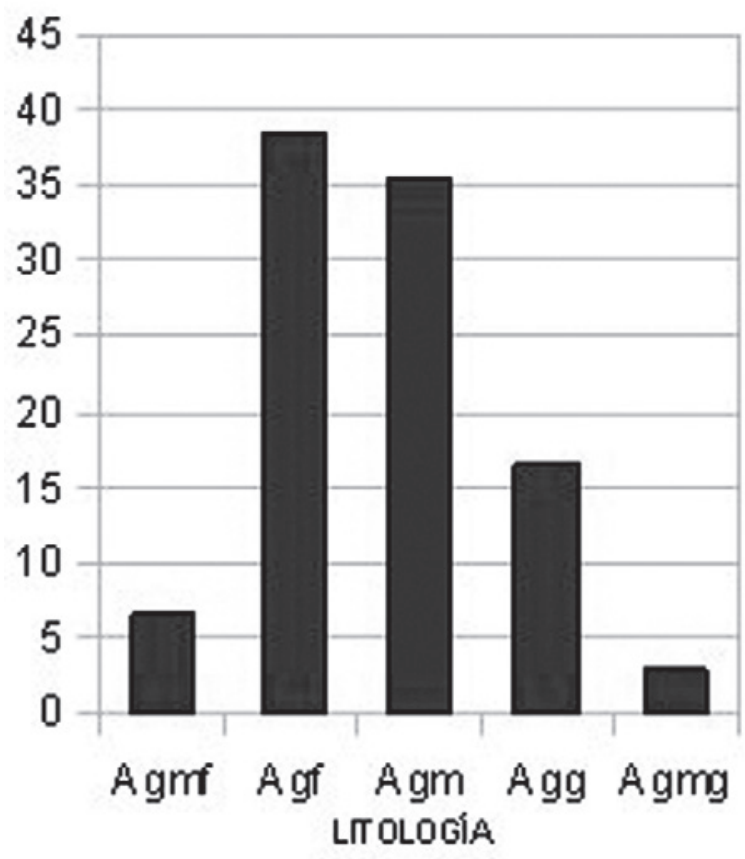

Figura 3. Distribución porcentual de tamaños de grano en los restos materiales detríticos de Los Partichuelos (Agmf: areniscas de grano muy fino; Agf: areniscas de grano fino; Agm: areniscas de grano medio; Agg: areniscas de grano grueso; Agmg; areniscas de grano muy grueso).

erosión, lo que satisfaría el criterio de selección de bases naturales compactas y duras en estos sedimentos.

Los escasos restos materiales elaborados a partir de cantos rodados de sílex o de metacuarcita, tendrían su área fuente en las terrazas fluviales de los cercanos ríos Guadarranque y Guadiaro. La cuenca fluvial de este último río comprende afloramientos de rocas metamórficas (inexistentes en la comarca) y de calizas jurásicas subbéticas que contienen sílex.

\section{LOS MODELOS DE REDUCCIÓN LÍTICAY CONFIGURACIÓN DE LAS HERRAMIENTAS DE TRABAJO A FINALES DEL MODO 2}

El estudio de los modelos de reducción lítica y de configuración de las herramientas de trabajo nos permite inferir las características morfotécnicas de las industrias líticas para comprender los procesos de trabajo desde la localización y selección de las materias primas, los procesos de reducción y de fabricación de las herramientas de trabajo, su utilización y, finalmente, su abandono. Solo después de un análisis detallado de este proceso, podremos determinar las características de un modelo comarcal, que nos sirva para su contrastación con otros modelos regionales para una época concreta.

Para este estudio hemos utilizado la información recabada de los sitios de Guadalquitón-Borondo-1 (San Roque, Cádiz), Guadalquitón-Borondo-4 (San Roque, Cádiz), Cortijo Carrasco (San Roque-La Línea de la Concepción, Cádiz), y Los Partichuelos (La Línea de la Concepción, Cádiz). Desestimando los sitios de Gudalquitón-Borondo (San Roque, Cádiz), Guadalquitón-Borondo-2 (San Roque, Cádiz), Guadalquitón-Borondo-3 (San Roque, Cádiz), La Alcaidesa (San Roque, Cádiz), Punta Mala (La Línea de la Concepción, Cádiz), Torre Nueva (La Línea de la Concepción, Cádiz) y Ringo Trinchera-1 (Los Barrios, Cádiz), ante el escaso número de evidencias arqueológicas localizadas, pero que sí son interesantes dentro de un estudio geológico y geomorfológico.

Estos sitios han proporcionado, como ya hemos tenido ocasión de comprobar, la masiva selección y aprovisionamiento de una materia prima como es la arenisca, especialmente aquellas bien cementadas (por sílice principalmente) y compactas (mayoritariamente de grano fino y medio) que ante los proceso de fracturación permiten obtener unos filos cortantes. El uso masivo de esta materia prima no sólo es consecuencia de su abundancia en el Campo de Gibraltar y de la escasa presencia del sílex, sino también por las características físico-químicas que presentan.

Estas areniscas seleccionadas en la fabricación de las herramientas de trabajo presentan un carácter local de adquisición inmediata, ya que aparecen en forma de Bna en el propio depósito donde se documentan los productos arqueológicos. Estas Bna seleccionadas para los procesos de reducción lítica y fabricación de las herramientas de trabajo se caracterizan igualmente, en relación a épocas anteriores, con una disminución de los volúmenes y los pesos de las materias primas, circunstancia que debe ponerse en relación con un aumento significativo de las herramientas sobre BP de tamaño mediano/pequeño y una disminución paulatina de los macroútiles (bifaces, triédros y cantos tallados) tanto sobre BN1G como sobre BP.

El estudio del rodamiento nos ha permitido identificar como los grados medio y poco son los más representativos, mientras que el grado bastante aparece en proporciones ínfimas; la pátina se constata en buena parte de los productos analizados. Estos datos son el resultado del tipo de depósito geológico donde se localizan (terrazas marinas) y su situación al aire libre. 
Cuadro 2. División porcentual de categorías estructurales por sitios arqueológicos.

\begin{tabular}{|c|c|c|c|c|c|}
\hline Sitio & BN1GE & BN1GC & BP & BN2G & TOTAL \\
\hline Guadalquitón-Borondo-1 & $\begin{array}{c}12 \\
\left(31^{\prime} 58 \%\right)\end{array}$ & $\begin{array}{c}1 \\
\left(2^{\prime} 63 \%\right)\end{array}$ & $\begin{array}{c}18 \\
\left(47^{\prime} 37 \%\right)\end{array}$ & $\begin{array}{c}7 \\
\left(18^{\prime} 42 \%\right)\end{array}$ & 38 \\
\hline Guadalquitón-Borondo-4 & $\begin{array}{c}10 \\
\left(26^{\prime} 32 \%\right)\end{array}$ & $\begin{array}{c}26 \\
\left(68^{\prime} 42 \%\right)\end{array}$ & $\begin{array}{c}2 \\
\left(5^{\prime} 26 \%\right)\end{array}$ & 38 \\
\hline Los Partichuelos & $\begin{array}{c}16 \\
\left(19^{\prime} 41 \%\right)\end{array}$ & - & $\begin{array}{c}163 \\
\left(68^{\prime} 78 \%\right)\end{array}$ & $\begin{array}{c}28 \\
\left(11^{\prime} 81 \%\right)\end{array}$ & 237 \\
\hline Cortijo Carrasco & $\begin{array}{c}78 \\
\left(27^{\prime} 74 \%\right)\end{array}$ & $\begin{array}{c}7 \\
\left(2^{\prime} 19 \%\right)\end{array}$ & $\begin{array}{c}20 \\
\left(15^{\prime} 67 \%\right)\end{array}$ & 137 \\
\hline
\end{tabular}

El estudio de las BN1G, dividido en BN1G de explotación (BN1GE) y de configuración (BN1GC), según el momento de la cadena lítica donde se encuentre, nos ha permitido identificar un dominio casi absoluto de las primeras; mientras que las BN1GC, que se documentan en proporciones ínfimas, tan sólo aparecen representadas por algunos cantos tallados (Cuadro 2). Así, se constata el dominio de los Temas Operativos Técnicos Indirectos (TOTI), y la presencia residual de los Temas Operativos Técnicos Directos (TOTD), en las estrategias de talla y configuración, que determinan el abandono de los grandes formatos y la generalización y estandarización de los medios y pequeños con una elevada elaboración.

Los TOTI están representados en cada uno de los sitios estudiados por (Cuadro 3):

El análisis de los TOTI nos permite inferir como frente a los modelos de gestión de las materias primas más simples, tales como las BN1GE relacionadas con una talla aleatoria no jerarquizada, y los modelos de gestión asociados con un esquema racional de la materia prima (longitudinal, bipolar ortogonal y bipolar opuesto), sobresalen, a excepción del caso del sitio de Los Partichuelos, los modelos de gestión caracterizados por una preparación de las Bna, exhaustividad en las extracciones y predeterminación de los productos obtenidos, relacionados fundamentalmente con los caracteres multipolar y centrípeto. Igualmente, destacar como en el grupo de los centrípetos algunos presenta un acondicionamiento parcial (Fig. 4).

En general, podemos comprobar como en los sitios estudiados las estrategias de reducción lítica están dominadas por aquellas que presentan un carácter progresista, frente a las técnicas de talla más simple. Todo ello nos informa de lo que posteriormente será el modo 3.

Las modificaciones y las estrategias observadas en los procesos de reducción lítica determinan las BP documentadas, las cuales están representadas por la siguiente división porcentual (Cuadro 4):

Las BP se ven representadas mayoritariamente por una homogeneidad dimensional donde dominan las de formato mediano/pequeño y la dimensión anchura, como consecuencia no sólo del tipo de materia prima utilizada sino también por las estrategias de reducción lítica

Cuadro 3. División porcentual de los distintos TOTI.

\begin{tabular}{|c|c|c|c|c|c|c|}
\hline Sitio & $\begin{array}{c}\text { Aleatorio } \\
\text { no jerarquizado }\end{array}$ & Longitudinal & $\begin{array}{l}\text { Bipolar } \\
\text { ortogonal }\end{array}$ & $\begin{array}{l}\text { Bipolar } \\
\text { opuesto }\end{array}$ & Multipolar & Centrípeto \\
\hline $\begin{array}{c}\text { Guadalquitón- } \\
\text { Borondo-1 }\end{array}$ & $\begin{array}{c}4 \\
\left(33^{\prime} 34 \%\right)\end{array}$ & $\begin{array}{c}1 \\
\left(8^{\prime} 33 \%\right)\end{array}$ & $\begin{array}{c}1 \\
\left(8^{\prime} 33 \%\right)\end{array}$ & & $\begin{array}{c}5 \\
\left(41^{\prime} 67 \%\right)\end{array}$ & $\begin{array}{c}1 \\
\left(8^{\prime} 33 \%\right)\end{array}$ \\
\hline $\begin{array}{l}\text { Guadalquitón- } \\
\text { Borondo-4 }\end{array}$ & $\begin{array}{c}1 \\
\left(10^{\prime} 00 \%\right)\end{array}$ & $\begin{array}{c}4 \\
\left(40^{\prime} 00 \%\right)\end{array}$ & & & $\begin{array}{c}5 \\
\left(50^{\prime} 00 \%\right)\end{array}$ & \\
\hline Los Partichuelos & $\begin{array}{c}7 \\
\left(15^{\prime} 21 \%\right)\end{array}$ & $\begin{array}{c}20 \\
\left(43^{\prime} 47 \%\right)\end{array}$ & $\begin{array}{c}3 \\
(6,52 \%)\end{array}$ & $\begin{array}{c}3 \\
\left(6^{\prime} 52 \%\right)\end{array}$ & $\begin{array}{c}9 \\
\left(19^{\prime} 57 \%\right)\end{array}$ & $\begin{array}{c}4 \\
\left(10^{\prime} 88 \%\right)\end{array}$ \\
\hline Cortijo Carrasco & $\begin{array}{c}6 \\
\left(15^{\prime} 79 \%\right)\end{array}$ & $\begin{array}{c}4 \\
\left(10^{\prime} 52 \%\right)\end{array}$ & & $\begin{array}{c}1 \\
\left(2^{\prime} 63 \%\right)\end{array}$ & $\begin{array}{c}21 \\
\left(55^{\prime} 27 \%\right)\end{array}$ & $\begin{array}{c}6 \\
\left(15^{\prime} 79 \%\right)\end{array}$ \\
\hline
\end{tabular}






Figura 4. Los Partichuelos. BN1GE: longitudinal (1), multipolar (2) y centrípeto (3). 
Cuadro 4. División porcentual de las distintas BP.

\begin{tabular}{|c|c|c|c|c|}
\hline Sitio & Cortical & Cortical/no cortical & No cortical/ cortical & No cortical \\
\hline Guadalquitón-Borondo-1 & $\begin{array}{c}1 \\
\left(5^{\prime} 56 \%\right)\end{array}$ & $\begin{array}{c}1 \\
\left(5^{\prime} 56 \%\right)\end{array}$ & $\begin{array}{c}4 \\
\left(22^{\prime} 22 \%\right)\end{array}$ & $\begin{array}{c}12 \\
\left(66^{\prime} 66 \%\right)\end{array}$ \\
\hline Guadalquitón-Borondo-4 & $\begin{array}{c}4 \\
\left(11^{\prime} 53 \%\right)\end{array}$ & - & $\begin{array}{c}19 \\
\left(15^{\prime} 38 \%\right)\end{array}$ & $\begin{array}{c}19 \\
\left(73^{\prime} 09 \%\right)\end{array}$ \\
\hline Los Partichuelos & 6 & 23 & 132 \\
$\left(3^{\prime} 68 \%\right)$ & $\left(14^{\prime} 12 \%\right)$ & $\left(80^{\prime} 98 \%\right)$ \\
\hline Cortijo Carrasco & 11 & 4 & 52 \\
$\left(14^{\prime} 47 \%\right)$ & $\left(5^{\prime} 26 \%\right)$ & $\left(68^{\prime} 43 \%\right)$ \\
\hline
\end{tabular}

empleadas, que determinan unos rasgos de estandarización de las BP; la disminución del tamaño de las BP favorece una reducción del coste energético en su transporte y una mayor rentabilidad de las materias primas; el análisis del volumen permite identificar el dominio de las no muy voluminosas (larga-espesa y larga-plana), frente a las espesas; dominio del carácter no cortical, donde se incluyen un reducido número de aquellas que se relacionan con un modelo de explotación relacionado con un acondicionamiento previo de las extracciones, seguido de los caracteres no cortical/cortical, cortical, y, finalmente, cortical/no cortical; la cara talonar está dominada por los unifacetados, los rotos/abatidos y los no facetados, documentándose en proporciones reducidas los que se relacionan con los bifacetados y los multifacetados, constatándose su presencia sobre todo en Cortijo Carrasco, en relación con el número de centrípetos.

Dentro del proceso técnico de reducción lítica y fabricación de las herramientas de trabajo, existe una relación lógica entre las BP y las BN2GC, donde estas últimas están realizadas fundamentalmente sobre BP no corticales y de tamaño mediano/pequeño (Cuadro 5).
En estos momentos se constata un aumento significativo de las herramientas de trabajo realizadas sobre BP (raederas, raspadores, muescas, denticulados, retoque abrupto) (Fig. 5), y una ausencia significativa de los macroútiles.

La diversidad de productos retocados identificados en este momento histórico nos permite inferir una mayor diversificación de la cadena operativa como respuesta a una ampliación de los procesos de trabajo, y el desarrollo específico de una herramienta para cada una de las actividades. Estas transformaciones deben ponerse en relación con las necesidades económicas y sociales desarrolladas a partir de ahora (Fig. 6).

El estudio de los procesos de reducción lítica y de fabricación de las herramientas de trabajo de estos sitios a finales del modo 2, nos ayuda a inferir los procesos de cambio y transformación, que no deben ser considerados para nada rupturistas, relacionados con una progresiva estandarización y sistematización de los procesos de obtención de productos, no lineal y con la posible convivencia de diferentes modos tecnológicos, que nos ayudan a plantear la existencia de distintas

Cuadro 5. División porcentual de las distintas BN2GC.

\begin{tabular}{|c|c|c|c|c|c|c|}
\hline Sitio & Raedera & Raspador & Muesca & Denticulado & Ret. Abrupto & Hendedor \\
\hline $\begin{array}{c}\text { Guadalquitón- } \\
\text { Borondo-1 }\end{array}$ & $\begin{array}{c}3 \\
\left(42^{\prime} 88 \%\right)\end{array}$ & $\begin{array}{c}1 \\
\left(14^{\prime} 28 \%\right)\end{array}$ & $\begin{array}{c}1 \\
\left(14^{\prime} 28 \%\right)\end{array}$ & $\begin{array}{c}1 \\
\left(14^{\prime} 28 \%\right)\end{array}$ & - & $\begin{array}{c}1 \\
\left(14^{\prime} 28 \%\right)\end{array}$ \\
\hline $\begin{array}{c}\text { Guadalquitón- } \\
\text { Borondo-4 }\end{array}$ & $\begin{array}{c}1 \\
\left(50^{\prime} 00 \%\right)\end{array}$ & & $\begin{array}{c}1 \\
\left(50^{\prime} 00 \%\right)\end{array}$ & & & \\
\hline Los Partichuelos & $\begin{array}{c}5 \\
\left(17^{\prime} 86 \%\right)\end{array}$ & $\begin{array}{c}4 \\
\left(14^{\prime} 28 \%\right)\end{array}$ & $\begin{array}{c}5 \\
\left(17^{\prime} 86 \%\right)\end{array}$ & $\begin{array}{c}7 \\
\left(25^{\prime} 00 \%\right)\end{array}$ & $\begin{array}{c}7 \\
\left(25^{\prime} 00 \%\right)\end{array}$ & - \\
\hline Cortijo Carrasco & $\begin{array}{c}4 \\
\left(20^{\prime} 00 \%\right)\end{array}$ & $\begin{array}{c}2 \\
\left(10^{\prime} 00 \%\right)\end{array}$ & $\begin{array}{c}5 \\
\left(25^{\prime} 00 \%\right)\end{array}$ & $\begin{array}{c}7 \\
\left(35^{\prime} 00 \%\right)\end{array}$ & $\begin{array}{c}2 \\
\left(10^{\prime} 00 \%\right)\end{array}$ & - \\
\hline
\end{tabular}




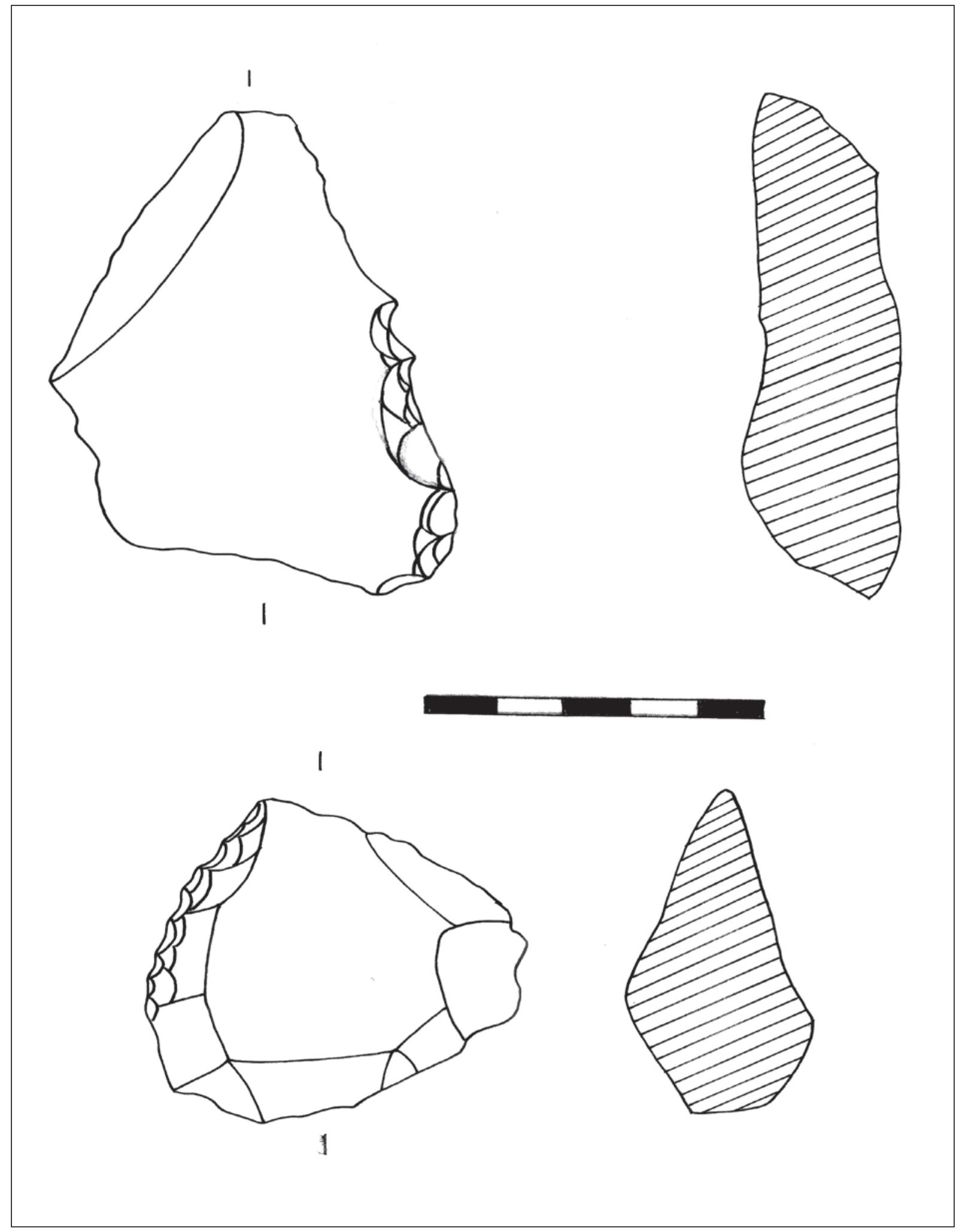

Figura 5. Cortijo Carrasco. BN2GC. Raederas. 


\section{CORTIJO CARRASCO}

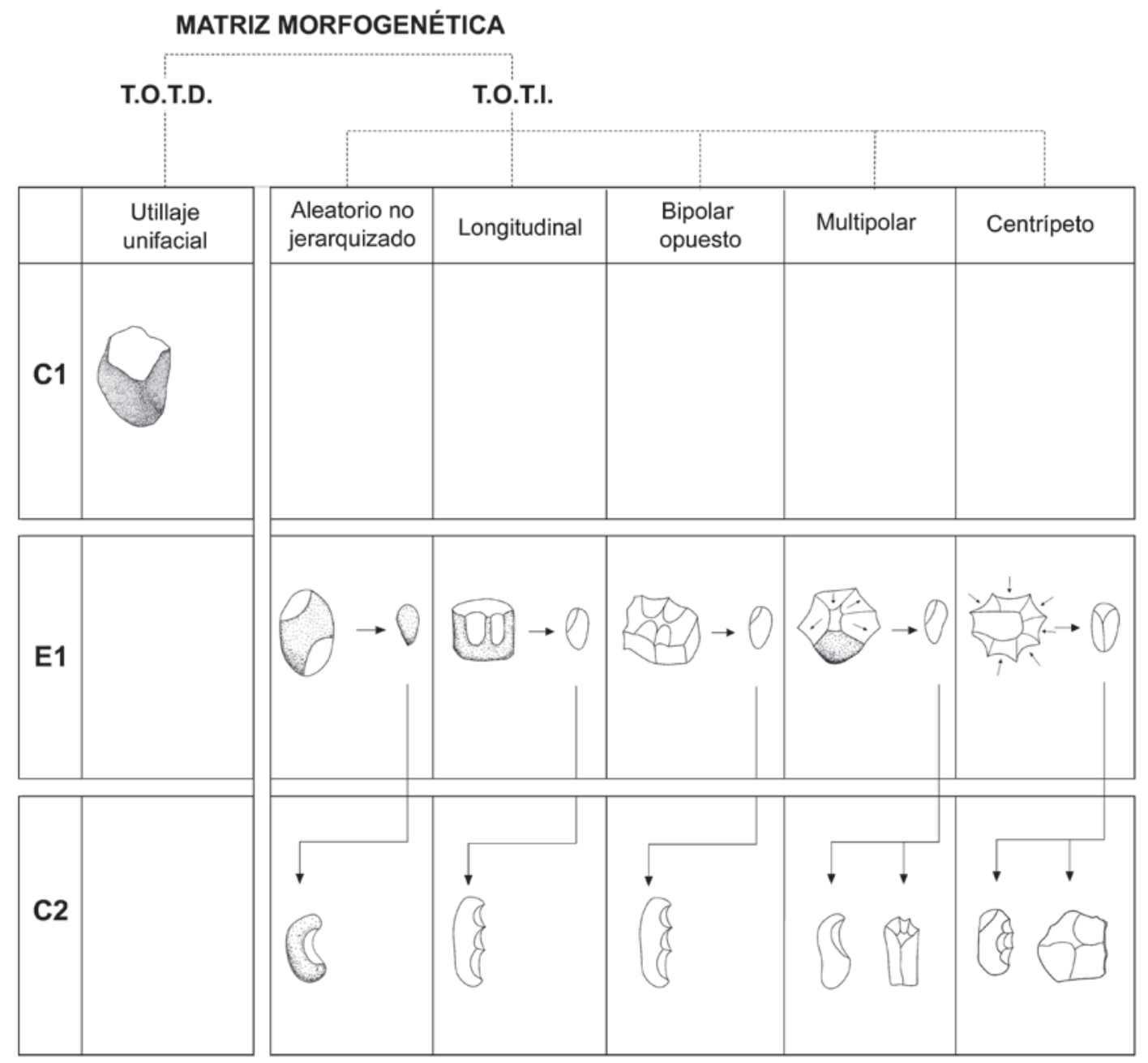

Figura 6. Matriz morfogenética del sitio de Cortijo Carrasco (San Roque-La Línea de la Concepción, Cádiz).

tradiciones regionales (variabilidad tecnológica) de desarrollo hacia el modo 3 atendiendo al substrato precedente existente en cada territorio (Vallespí 1992).

\section{EL FINAL DEL MODO 2 EN EL CAMPO DE GIBRALTAR Y SU CONTEXTUALIZACIÓN HISTÓRICA EN EL EXTREMO SUR DE LA PENÍNSULA IBÉRICA}

Las modificaciones observadas en los procesos de reducción lítica y de configuración de las herramientas de trabajo a finales del modo 2 , deben ponerse en relación con una serie de transformaciones en el ámbito biosocial, que repercuten, entre otros aspectos, en una nueva articulación social del territorio. Así, a partir de este momento histórico podemos comprobar cómo estas sociedades no sólo habitan los biotopos relacionados con los principales ámbitos fluviales del Campo de Gibraltar (ríos Palmones, Guadarranque, Guadiaro y Hozgarganta) en sus tramos de costa y valle como había ocurrido en buena parte del modo 2 (Castañeda, coord. 2008)(Fig. 7), sino también, desde estas vías naturales de comunicación con las zonas de montaña, aunque 


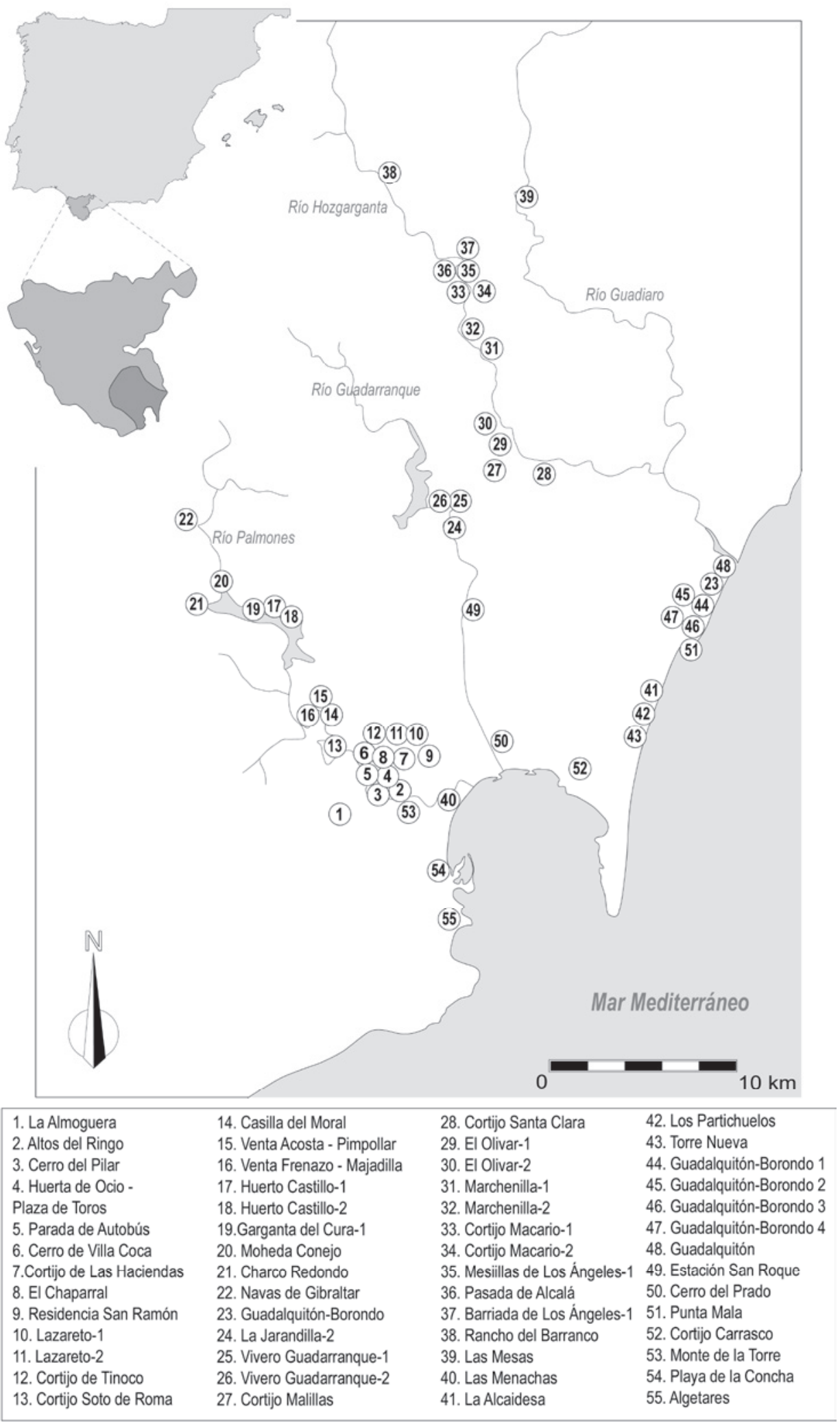

Figura 7. Localización geográfica de los sitios relacionados con el modo 2 en el Campo de Gibraltar. 


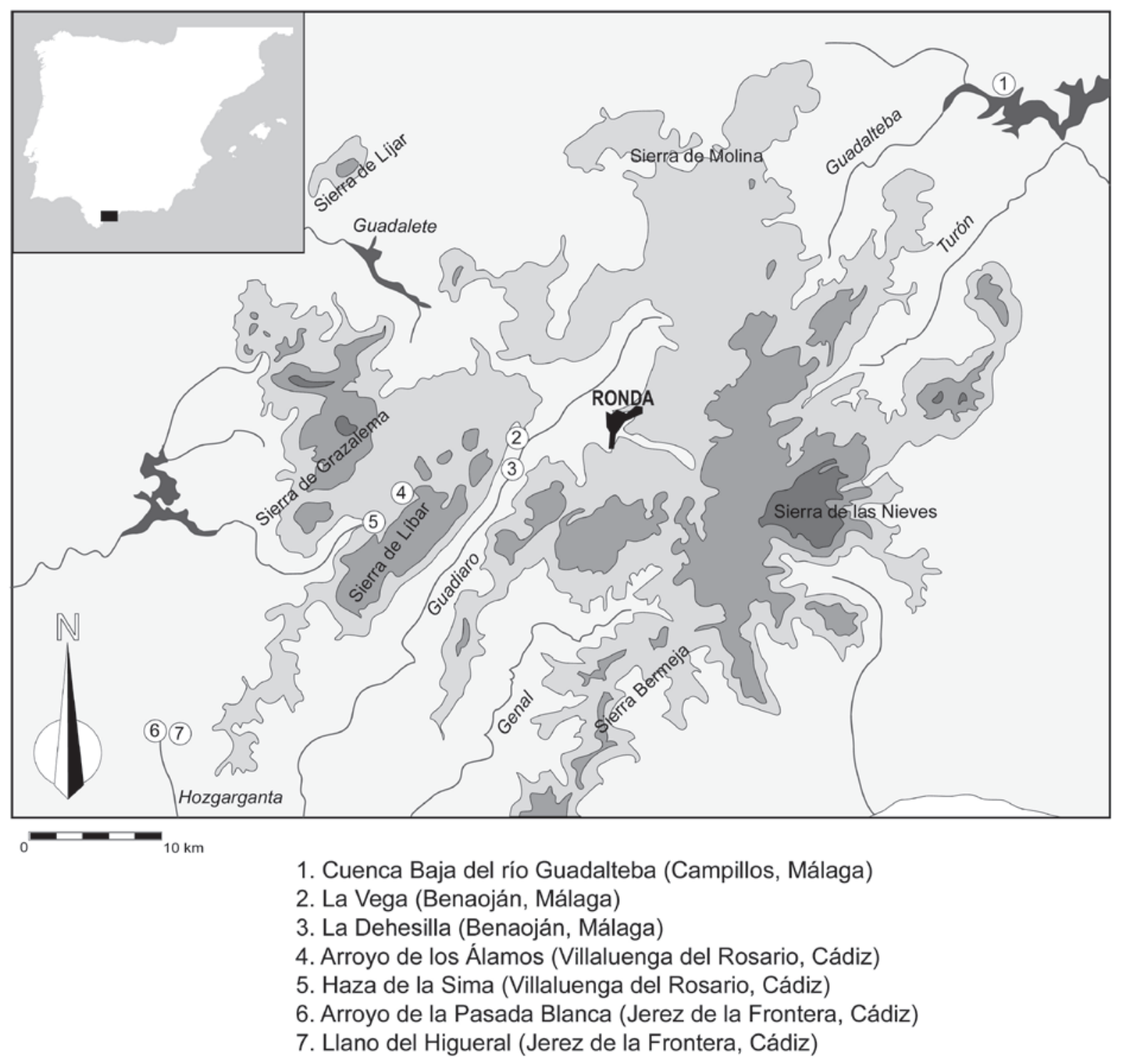

Figura 8. Localización geográfica de los sitios relacionados con las últimas etapas del modo 2 en la serranía de Ronda.

siempre relacionados con los ámbitos fluviales y sus zonas limítrofes (incluidos los medios kárstico asociados a ellos), no habitando aún las zonas más agrestes. Estas transformaciones se producen en distintos sitios del ámbito peninsular atendiendo a los diferentes modelos regionales, y en particular en la comarca del Campo de Gibraltar a finales del modo 2, relacionado con un calentamiento global y probablemente asociado al inicio del subestadio isotópico 5e (130-122 Ka).

Los valles fluviales del Campo de Gibraltar no solamente se convierten en unos lugares idóneos para la vida de estas sociedades, al ofrecer unos recursos variados como consecuencia de localizarse en entornos ecológicos diversificados; sino también en vías naturales de comunicación que relacionan diversos espacios geográficos. Así, el río Palmones (Castañeda, coord., 2008) favorece la comunicación con los principales ámbitos fluviales de la banda atlántica de Cádiz (Giles et al. 1993; Ramos 2008; Santiago et al. 2001) y de la Baja Andalucía; mientras, que por otra parte, los ríos Guadiaro y Hozgarganta permiten una comunicación no sólo con las zonas de costa y de valle, sino también de montaña, y especialmente con el área de la serranía de Ronda, en los momentos finales del modo 2 
(Castañeda 2009). A partir de estos sitios localizados en la comarca del Campo de Gibraltar y de otros (Guadalete, Corbones o Guadalhorce) se consolida definitivamente el primer poblamiento humano en la zona más occidental de las cordilleras Béticas. Este hecho se constata a finales del modo 2, en sitios tales como Haza de la Sima (Villaluenga, Cádiz)(Giles, et al. 2003), Arroyo de los Álamos (Villaluenga, Cádiz)(Giles et al. 2003), La Vega (Benaoján, Málaga)(Becerra y Cantalejo 2002), La Dehesilla (Benaoján, Málaga)(Becerra y Cantalejo 2002), Llano del Higueral (Jerez de la Frontera, Cádiz) (Giles et al. 2003), entre otros (Fig. 8). Estos sitios de la sierra se caracterizan por unos modelos de reducción lítica y de fabricación de las herramientas de trabajo que avanzan lo que posteriormente será el modo 3, tales como una mayor preocupación por la selección de las materias primas, donde se constata un dominio absoluto del sílex; generalización de unos esquemas racionales y unas estrategias complejas en la gestión de las materias primas (centrípeto); una disminución de los TOTD (bifaces, triédros y cantos tallados); y un aumento significativos de las herramientas sobre BP muy elaboradas.

Las modificaciones observadas en las estrategias de gestión y reducción de las materias primas, y la consiguiente configuración de las herramientas de trabajo; la nueva articulación social del territorio, con la llegada de estos homínidos a las zonas de montaña; y los cambios observados en el ámbito biosocial, podría relacionarse cronológicamente con el calentamiento global generalizado observado a inicios del subestadio isotópico 5e (130-122 ka). Este proceso, empieza a definirse en el Sur de la Península Ibérica gracias a las evidencias materiales localizadas en sitios tales como la cueva del Ángel (Lucena, Córdoba) (Botella et al. 2006), situada a 620 m.s.n.m., que ha ofrecido una cronología de $121 \pm 10 \mathrm{ka}$ por U/Th (OIS5) para las últimas etapas del modo 2; o la localizada en la cueva de la Carihuela (Piñar, Granada), en un farallón rocoso en plena Sierra de Harana a 1000 m.s.n.m, donde su nivel basal adscrito también a las últimas etapas del modo 2 presenta unas dataciones entre 146 y 117 ka (Vega Toscano 1988, 1997). Igualmente, en este contexto podríamos mencionar, aunque carezcan actualmente de dataciones absolutas, pero si con buenos registros faunísticos, los sitios de la Solana del Zamborino (Fonelas, Granada) (Botella et al. 1976; Martínez y López Reyes 2001) $\mathrm{y}$, un poco anterior a este último, Cueva Horá (Darro, Granada)( Botella et al. 1983).

La nueva articulación social del territorio, y su ocupación por medio del nomadismo, debe ser explicado junto a la búsqueda de nuevas materias primas como el sílex (Vallespí 1992) y a la elaboración de unas herramientas más específicas, con una nueva realidad social y unos comportamientos que nos acercan a la forma de vida de Homo neanderthalensis clásico.

\section{BIBLIOGRAFÍA}

BECERRA, M. y CANTALEJO, P. (2002): "Yacimiento de La Vega. Importante hallazgo de industria lítica perteneciente al Paleolítico Inferior en Benaoján”, Benaoján Mensual 28: 12. Málaga.

BOTELLA, D.; BARROSO, C.; RIQUELME, J.A.; ABDESSADOK, S.; CAPARRÓS, M.; VERDÚ, L.; MONGE, G. y GARCÍA, J.A. (2006): "La Cueva del Ángel (Lucena, Córdoba), un yacimiento del pleistoceno medio y superior del sur de la Península Ibérica", Trabajos de Prehistoria 63 (2): 153-165. Madrid.

BOTELLA, M.C.; VERA, J.A.; PORTA, J.; CASAS, J.; PEÑA, J.A.; MARQUEZ, I.; BENITO, A.; RUIZ, A. y DELGADO, M.T. (1976): “El yacimiento achelense de la Solana del Zamborino, Fonelas (Granada). Primera campaña de excavaciones", Cuadernos de Prehistoria 1: 1-45. Granada.

BOTELLA, M.C.; MARTÍNEZ, C.; CÁRDENAS, F. y CAÑABATE, M.J. (1983): "Las industrias paleolíticas de Cueva Horá (Darro, Granada). Avance al estudio técnico y tipológico", Antropología y $\mathrm{Pa}$ leoecología Humana 3: 13-48. Granada.

CARBONELL, E. (1982): Application de la méthode dialectique à la construction d'un système anlytique pour l'étude des matériaux du Paléolithique inférieur. Dialektiké deTypologie Analitique. Inst. Universitaire de Recherche Scientifique.

CARBONELL, E; GUILBAUD, M. y MORA, R. (1983): "Utilización de la lógica analítica para el estudio de tecno-complejos a cantos tallados", Cahier Noir 1: 3-64.

CARBONELL, E., RODRIGUEZ, X.P.; SALA, R. y VAQUERO, M. (1992): "New elements of the logical analytic system", Cahier Noir 6: 3-59.

CASTANEDA, V., coord. (2008): Las primeras ocupaciones humanas de los Barrios (Cádiz). El ejemplo proporcionado por el río Palmones, Servicio de Publicaciones de la Universidad de Cádiz e Istmo, Ayuntamiento de la Villa de Los Barrios, Cádiz.

CASTAÑEDA, V. (2009): "El primer poblamiento humano de la serranía de Ronda y su relación con el Campo de Gibraltar. Una aproximación histórica" Mainake XXX: 331-344, Diputación de Málaga, Málaga.

CASTAÑEDA, V.; HERRERO, N.; MARISCAL, D.; TORRES, F.; DOMÍNGUEZ-BELLA, S. y 
CASTAÑEDA, A. (2005): "Las bandas de cazadores-recolectores en el Campo de Gibraltar. Balance tras nuestra primera campaña de prospección superficial en el termino municipal de Los Barrios (Cádiz)", Anuario Arqueológico de Andalucía. 2002. II Actividades Sistemáticas y Puntuales: 9-21, Consejería de Cultura, Junta de Andalucía.

CASTAÑEDA, V.; TORRES, F.L.; COSTELA, Y.; PÉREZ, L. y BERNAL, J.M. (2008): "Las primeras ocupaciones humanas de San Roque. Las sociedades del Paleolítico", $L A C Y, 0$, Revista de estudios Sanroqueños: 9-24. San Roque, Cádiz.

CASTAÑEDA, V.; PÉREZ, L.; TORRES, F.; COSTELA, Y.; JIMÉNEZ-CAMINO, R. y TOMASSETTI, J.M. (en prensa a): "Los modelos de reducción lítica en Algetares (Algeciras, Cádiz) durante el modo 2 y su contextualización histórica en el ámbito del Estrecho de Gibraltar", Espacio, Tiempo y Forma, 2. Serie I, Prehistoria y Arqueología, Nueva Época, UNED. Madrid.

CASTAÑEDA, V.; COSTELA, Y.; TORRES, F. y PÉREZ, L. (en prensa b): "Ventorrillo de la Trocha 1 y 2 (Algeciras, Cádiz). La consolidación de la economía de producción en el Campo de Gibraltar durante el IV y el III milenio a.n.e", II Jornadas de Prehistoria y Arqueología en el Campo de Gibraltar. Los Barrios 5, 6 y 7 de junio de 2009. Cádiz.

FINLAYSON, C. y GILES, F. (1999): “The southern Iberian Peninsula in the Late Pleistocene: Geographie, Ecology and Human occupation", en C.B. STRINGER, R.N.E. BARTON y J.C. FINLAYSON (eds.), Gibraltar and the Neanderthals 18481998: 139-154. Oxford.

GAVALA, J. (1924): "Mapa Geológico de la provincia de Cádiz, escala 1:200.000". I.G.M.E.

GILES, F.; GUTIÉRREZ, J.M.; SANTIAGO, A.; MATA, E. y GRACIA, F.J. (1993): "Prospecciones arqueológicas y análisis geocronológicos y sedimentológicos en la cuenca del río Guadalete", Investigaciones Arqueológicas en Andalucía, 19851992: 211-218. Huelva.

GILES, F.; GRACIA, F.J.; SANTIAGO, A.; GUTIÉRREZ, J.M.; MATA, E.; AGUILERA, L.; FINLAYSON, C.; PIÑATEL, F. y BARTON, N. (2000): "Nuevas aportaciones a los complejos tecnológicos del Pleistoceno medio y superior del Campo de Gibraltar. Los yacimientos de El Chaparral (Los Barrios) y Guadalquitón-Borondo (San Roque)", Caetaria 3: 13-26, Museo Municipal de Algeciras, Algeciras.

GILES, F.; SANTIAGO, A.; AGUILERA, L.; GUTIÉRREZ, J.M. y FINLAYSON, C. (2003): "Paleolítico
Inferior y Medio en la Sierra de Cádiz. Evidencias de grupos de cazadores-recolectores del Pleistoceno Medio y Superior", Almajar 1: 8-35, Villamartín. GOY, J.; ZAZO, C.; SILVA, P.; LARIO, J.; BARDAJÍ, T. y SOMOZA, L. (1995): "Evaluación geomorfológica del comportamiento geotectónico del Estrecho de Gibraltar (Zona Norte) durante el cuaternario", en M. Esteras (coord.), IV Coloquio Internacional sobre el Enlace Fijo del Estrecho de Gibraltar. SE$C E G$, Vol. II: 51-69. Madrid.

LARIO, J. (1996): Último y Presente Interglacial en el área de conexión Atlántico-Mediterráneo (Sur de España). Variaciones del nivel del mar, paleoclima y paleoambientes: 1-269. Tesis Doct. Universidad Complutense. Madrid.

MARTÍN-ALGARRA, A. (1987): "Evolución geológica alpina del contacto entre las zonas internas y las zonas externas de la Cordillera Bética”. 1-1.171. Tesis Doctoral Univ. Granada.

MARTÍNEZ, G. y LÓPEZ, V. (2001): "La Solana del Zamborino", Paleontología i Evolució 32-33: 2330. Barcelona.

PENDÓN, J., (1978): Sedimentación turbidítica en las unidades del Campo de Gibraltar, Tesis Doctoral. Secretariado de Publicaciones de la Universidad de Granada.

RAMOS, J. (2008): "Novedades en el estudio de los grupos humanos portadores del tecnocomplejo musteriense-Modo III en la banda atlántica de Cádiz, en el contexto del Estrecho de Gibraltar", Homenaje a Ignacio Barandiarán Maestu. Veleia 2425: 397-414. Universidad del País Vasco.

RODRIGUEZ-VIDAL, J.; CÁCERES, L.; GRACIA, F.; MARTINEZ, F.; FINLAYSON, C.; GILES, F.; SANTIAGO, A. y PEGUERO, C. (2002): "El relieve kárstico de Gibraltar como registro morfosedimentario durante el Cuaternario (Mediterráneo occidental)", SEDECK 3: 6-15.

RUIZ BUSTOS, A. (1991):’Hacia una cronología del Cuaternario continental ibérico. Cuestiones básicas a considerar", Raña-AEQUA 10: 45-51.

RUIZ BUSTOS, A., (1997): “Características bioestratigráficas y paleoecológicas que implican los mamíferos cuaternarios en las cuencas de la cordillera Béticas", Cuaternario Ibérico: 283-296. Huelva.

RUIZ-REIG, P. (1994): "Mapa y memoria de la Hoja no 87 (Algeciras) a escala 1:200.000”. I.G.M.E.

SÁNCHEZ GOÑI, M.F. y d'ERRICO, F. (2005): "La historia de la vegetación y el clima del último ciclo climático (OIS5-OIS1, 140.000-10.000 años BP) en la Península Ibérica y su posible impacto sobre los grupos paleolíticos", en R. Montes, y J.A. Laceras 
(coords.), Neandertales Cantábricos, Estado de la Cuestión. Monografías, 20: 115-129. Museo Nacional y Centro de Investigaciones de Altamira, Santander.

SANTIAGO, A.; GUTIÉRREZ, J.M.; GILES, F.; MATA, E. y AGUILERA, L. (2001): "El registro arqueológico de los primeros grupos humanos en la comarca de Jerez de la Frontera y su contexto en el sur de la Península Ibérica. Resultados de un proyecto de Investigación", Revista de Historia de Jerez 7. Cuaderno de Arqueología. Jerez de la Frontera.

VALLESPÍ, E. (1992): “Las industrias achelenses de Andalucía: ordenación y comentarios”, Spal 1: 61-78. Universidad de Sevilla. http://dx.doi.org/10.12795/ spal.1992.i1.03

VEGA TOSCANO, L.G. ; HOYOS, M. ; RUIZ BUSTOS, A. y LAVILLE, H. (1988): "La séquence de la grotte de la Carihuela (Piña, Grenada): Cronostratigraphie et paléolécologie du Pléistocène supérieur au sud de la Péninsule Ibérique", en L'Homme de
Neandertal. Vol. 2: l'Environnement: 169-180. Université de Liége.

VEGA TOSCANO, L.G.; COSANO, P.; VILLAR, A.; ESCARPA, O. y ROJAS, T. (1997): "Las industrias de la interfase Pleistoceno Medio-Superior en la cueva de la Carihuela (Piñar, Granada)", II Congreso de Arqueología Peninsular. Vol. I: Paleolítico y Epipaleolítico: 105-119. Zamora.

ZAZO, C.; GOY, J.; HILLAIRE, C.; HOYOS, M.; CUERDA, J.; GHALEB, B.; BARDAJÍ, T.; DABRIO, J.; LARIO, J.; SILVA, P.; GONZÁLEZ, A.; GONZALEZ, F. y SOLER, V. (1997): "El nivel del mar y los interglaciales cuaternarios: $\mathrm{Su}$ registro en las costas peninsulares e insulares españolas", Cuaternario Ibérico. AEQUA monografías: 23-32.

ZAZO, C.; SILVA, P.; GOY, J.; HILLAIRE, C.; GHALEB, B.; LARIO, J.; BARDAJÍ, T. y GONZÁLEZ, A. (1999): "Coastal uplift in continental colision plate boundaries: data from Last Interglacial marine terraces of the Gibraltar Strait area (south Spain)", Tectonophysics 301: 95-109.

FeCHA DE ENTRADA: 18-11-2009

FECHA DE ACEPTACIÓN: 22-12-2009 